\title{
Early detection of prostate cancer local recurrence by urinary prostate-specific antigen
}

\author{
Stéphane Bolduc, MD; ${ }^{*}$ Brant A. Inman, MD; ${ }^{\dagger}$ Louis Lacombe, MD; ${ }^{*}$ Yves Fradet, MD; \\ Roland R. Tremblay, MD, $P h D^{\dagger}$
}

See related article on page 218

\begin{abstract}
Purpose: We assessed the role of urinary prostate-specific antigen (uPSA) in the follow-up of prostate cancer after retropubic radical prostatectomy (RRP) for the early detection of local recurrences. Methods: We recruited 50 patients previously treated for prostate cancer with RRP and who had not experienced a prostatespecific antigen (PSA) recurrence within their first postoperative year into a cross-sectional laboratory assessment and prospective 6-year longitudinal follow-up study. We defined biochemical failure as a serum PSA (sPSA) of $0.3 \mu \mathrm{g} / \mathrm{L}$ or greater. Patients provided blood samples and a 50-mL sample of first-voided urine. We performed Wilcoxon rank-sum and Fisher exact tests for statistical analysis.
\end{abstract}

Results: The median sPSA was $0.13 \mu \mathrm{g} / \mathrm{L}$. The median uPSA was $0.8 \mu \mathrm{g} / \mathrm{L}$, and was not significantly different when comparing Gleason scores or pathological stages. Of the 50 patients, 27 initially had a nondetectable sPSA but a detectable uPSA, and 11 patients experienced sPSA failure after 6 years. Six patients had detectable sPSA and uPSA initially. Fifteen patients were negative for both sPSA and UPSA, and 13 remained sPSA-free after 6 years. The odds ratio (OR) of having sPSA failure given a positive UPSA test was 4.5 if sPSA was undetectable, but was reduced to 2.6 if sPSA was detectable. The pooled Mantel-Haenszel OR of 4.2 suggested that a detectable uPSA quadrupled the risk of recurrence, independent of whether sPSA was elevated or not. The sensitivity of uPSA for detecting future sPSA recurrences was $81 \%$ and specificity was $45 \%$.

Conclusion: Urinary PSA could contribute to an early detection of local recurrences of prostate cancer after a radical prostatectomy.

Can Urol Assoc J 2009;3(3):213-7

\section{Résumé}

Objectif : Nous avons évalué le rôle de I'antigène prostatique spécifique (APS) urinaire dans le suivi du cancer de la prostate après prostatectomie radicale rétropubienne (PRR) pour le dépistage précoce de récidives locales.

Méthodes : Cinquante patients atteints de cancer de la prostate traités par PRR et n'ayant présenté aucune récidive avec anomalie de I'APS dans l'année suivant l'intervention chirurgicale ont été inscrits à une étude transversale par épreuves de laboratoire avec suivi longitudinal prospectif sur 6 ans. L'échec sur le plan biochimique était défini comme un taux d'APS sérique de $0,3 \mu \mathrm{g} / \mathrm{L}$ ou plus. Les patients devaient fournir des échantillons de sang et un échantillon d'urine du matin de $50 \mathrm{~mL}$. Les analyses statistiques reposaient sur le test de Wilcoxon et la méthode exacte de Fisher.

Résultats : La valeur médiane de l'APS sérique était de 0,13 $\mu \mathrm{g} / \mathrm{L}$. La valeur médiane de l'APS urinaire était de $0,8 \mu \mathrm{g} / \mathrm{L}$; la différence était non significative quand on tenait compte des scores de Gleason ou des stades pathologiques. Sur les 50 patients, 27 présentaient des taux d'APS sérique non décelables au début, mais des taux d'APS urinaire décelables; 11 patients ont présenté un échec quant aux taux d'APS sérique après 6 ans. Six patients avaient des taux d'APS sérique et urinaire décelables au départ. Quinze patients n'avaient aucun taux décelable d'APS sérique ou urinaire, et aucun APS sérique n'était toujours décelable chez 13 patients après 6 ans. Le rapport de risque d'un échec quant aux taux d'APS sérique après détection d'APS urinaire est de 4,5 en I'absence d'un taux d'APS sérique décelable, mais diminue à 2,6 en présence d'un taux d'APS sérique décelable. Le rapport de risque cumulé de 4,21 calculé par la méthode de Mantel-Haenszel porte à croire que des taux d'APS urinaire décelables quadruplent le risque de présenter une récidive, que les taux sériques soient élevés ou non. La sensibilité du test de dépistage de I'APS urinaire pour la détection des récidives avec anomalie des taux sériques était de $81 \%$, et la spécificité, de $45 \%$. Conclusion : Le taux d'APS urinaire peut contribuer à un dépistage précoce des récidives locales après une prostatectomie radicale.

\section{Introduction}

In the past 10 years, emotional debates about new markers of prostate function and pathology have flooded the urological literature and stimulated investigators to deepen their knowledge about kallikreins and other prostate-derived molecules. Serum prostate-specific antigen (sPSA) has proved to be a useful marker in the diagnosis and management of prostate cancer. Graves and colleagues ${ }^{1}$ demonstrated that not only was prostate-specific antigen (PSA) found in the serum, but that it was also detectable in the urine, an 
observation that thus far has not received much attention. Urinary PSA (UPSA) has not been carefully examined as a marker of prostate cancer.

It has been hypothesized that uPSA might prove to be a useful monitor of local tumour recurrence after radical retropubic prostatectomy (RRP). ${ }^{2}$ Yet, there is no broad consensus among investigators about the particular role for UPSA, suggesting that heterogeneous methodology and technical issues may bias the results of uPSA studies. ${ }^{3}$ The method of collection of urine samples has varied considerably among studies, with specimens obtained before and after prostate massage, ${ }^{4}$ in first-voided specimens ${ }^{2,4,5}$ in midstream samples ${ }^{6-8}$ and in 24-hour collections. ${ }^{9,10}$ It is quite likely that differences in study methodology are contributing to contradictory results.

To differentiate post-RRP local recurrences from metastatic disease, it is helpful to examine tumour pathology and PSA dynamics. Several parameters, including the timing of PSA failure, PSA doubling time and PSA velocity may give clues as to the nature of the recurrence. ${ }^{11-13}$ None of these measures is wholly accurate, and potentially curable local recurrences can be misdiagnosed as metastatic disease and vice versa.

\begin{tabular}{|c|c|}
\hline Variable & $\begin{array}{l}\text { Measurement } \\
\text { no. (\%) of participants* }\end{array}$ \\
\hline Age, median, yr (IQR) & $64.5(60.3-70.0)$ \\
\hline \multicolumn{2}{|l|}{ TNM stage } \\
\hline T1A/B & $316)$ \\
\hline $\mathrm{T} 2$ & $25(50)$ \\
\hline T3 & $22(44)$ \\
\hline NO & 44 (88) \\
\hline $\mathrm{N} 1 / 2$ & $6(12)$ \\
\hline \multicolumn{2}{|l|}{ Gleason score } \\
\hline 6 & $36(72)$ \\
\hline 7 & $5(10)$ \\
\hline 8-10 & $9(18)$ \\
\hline \multicolumn{2}{|l|}{ Serum PSA } \\
\hline Median, $\mu \mathrm{g} / \mathrm{L}$ (IQR) & $0.5(0.0-0.0)$ \\
\hline$>0$ & $33(66)$ \\
\hline $\begin{array}{l}\text { Serum testosterone, nmol/L } \\
\text { (IQR) }\end{array}$ & $1.0(0.7-1.6)$ \\
\hline \multicolumn{2}{|l|}{ Follow-up events } \\
\hline PSA failure & $21(42)$ \\
\hline Metastases & 9 (18) \\
\hline Follow-up, median, yr (IQR) & $7.6(5.1-9.3)$ \\
\hline
\end{tabular}

After RRP, small amounts of UPSA are sometimes detectable and may be the product of androgen-sensitive glandular elements lining the urethra. Consequently, any patient who is androgen-deprived should not have a detectable uPSA. In fact, it has been suggested that one should seriously consider the possibility of a local cancer recurrence if the UPSA is elevated, rising rapidly, or if it is detectable in an androgen-deprived patient. The specific detection of a local recurrence may allow the application of effective local salvage therapy such as radiotherapy, which reduces the probability of progression to metastatic disease. The aim of the present study was to assess whether uPSA is capable of accurately detecting tumour recurrences after RRP.

\section{Methods}

\section{Patient selection}

We recruited a group of 50 men previously treated for prostate cancer with RRP who had not experienced an SPSA recurrence within their first postoperative year to participate in our study. We recruited patients consecutively during urological oncology clinical sessions in 2000. During these routine clinical visits, we conducted a crosssectional laboratory assessment of the consenting men with a planned prospective 1-year longitudinal follow-up period and a primary outcome of biochemical failure (defined as a single sPSA $\geq 0.3 \mu \mathrm{g} / \mathrm{L}$ ). Since the initial event-rate was lower than expected, we extended the study follow-up period by 5 years. Owing to potential interference with the UPSA test, we treated patients with active urinary tract infections and then reassessed them at a later date. We obtained approval from our institutional review board and written informed consent from all participants.

\section{Laboratory assessment}

Patients provided blood samples for the measurement of sPSA and bioavailable testosterone (BT). We measured BT to determine the active androgen level of every patient so that any biasing effect of androgen level on uPSA results could be assessed. We assayed BT using the ammonium sulfate radioimmunoassay method described by Tremblay and Dubé, ${ }^{14}$ whereas we measured sPSA using the Hybritech assay.

We measured uPSA in a 50-mL urine sample obtained without restriction on time of day. Since preliminary testing performed on participants with normal or hyperplastic prostate suggested that uPSA levels were optimally 
detected from 1 to 6 hours after the previous void and could be falsely elevated by sexual activity or prostatic massage,${ }^{9}$ we obtained all urine samples after a minimum 1 -hour period after the previous void, after a 24-hour period of sexual abstinence and before digital rectal examination. We stored urine samples at $-20^{\circ} \mathrm{C}$ until assayed. We measured uPSA levels using a sandwich enzyme-linked immunosorbent assay (ELISA) method with a polyclonal capture antibody (Poly PSA) and a monoclonal detection antibody 4D1 (developed in laboratory by R.R.T.). Readings occurred in a spectrophotometer at $414 \mathrm{~nm}$. The method has a detection limit of $0.3 \mu \mathrm{g} / \mathrm{L}$ and intra- and interassay coefficients of variation of less than $4 \%$. We blinded patients and their urologists from uPSA results to ensure that patient management decisions were not influenced by the uPSA results.

\section{Statistical methods}

We performed statistical analyses using R 2.5.0 (R Foundation for Statistical Computing). ${ }^{15}$ We compared the distributions of continuous and categorical variables using the Wilcoxon rank-sum test and Fisher exact test, respectively. We measured the association of positive uPSA tests with future biochemical failure with odds ratios (ORs) using the Yates method of zero cell adjustment. We stratified the ORs by sPSA category at baseline (detectable v. undetectable), and we calculated a pooled OR using the Mantel-Haenszel method. ${ }^{16}$ We constructed univariate Cox models to determine which clinical factors predicted biochemical failure. All $p$ values were 2 -sided and we considered them to be statistically significant at $p \leq 0.05$.

\section{Results}

The clinical characteristics of the patient cohort are detailed in Table 1. Briefly, the median patient age was 64.5 years and the median time from RRP to last follow-up was 7.6 years. Though most patients had an undetectable sPSA at baseline, $8(16 \%)$ had a detectable sPSA that had not yet reached our failure limit of $0.3 \mu \mathrm{g} / \mathrm{L}$. Biochemical failure eventually occurred in 21 patients (42\%), 9 in whom metastases later developed. Univariate Cox models showed that T3 tumour stage (hazard ratio [HR] 4.4, 95\% confidence interval [Cl] 1.7-11.2, $p=0.001$ ), positive lymph nodes (HR 6.5, 95\% Cl 2.4-17.5, $p=0.001$ ), and Gleason score (HR 1.5, 95\% Cl 1.2-1.9, $p=0.002$ ) predicted biochemical failure, whereas serum BT (HR 1.2, 95\% Cl 0.8-2.0, $p=0.41)$ and patient age (HR 1.0, 95\% Cl 0.9-1.1, $p=0.88$ ) did not. Two patients died from a different cancer and 11 were lost to follow-up after 5 years.
Of the 50 patients in the study, 27 had undetectable sPSA at baseline, whereas their uPSA levels were detectable. Of these, $11(41 \%)$ eventually experienced a sPSA failure and received treatment: 2 of 2 responded to radiotherapy, 4 of 4 responded to androgen deprivation therapy (ADT) and 5 are currently stable and under active surveillance. A total of 15 patients had undetectable sPSA and UPSA at baseline, 2 of whom experienced prostate cancer recurrence (1 responded to radiotherapy, 1 responded to ADT). Six patients had a detectable sPSA and UPSA at baseline (4 of 5 responded to radiotherapy, 1 of 5 failed radiotherapy and received ADT, and 1 received ADT). Lastly, 2 patients had a detectable sPSA with an undetectable uPSA at baseline and received ADT.

At baseline, 33 patients (66\%) had a detectable uPSA, 17 of whom (52\%) went on to experience prostate cancer recurrence over the following 5 years. We found that no factor, including tumour stage, presence of positive lymph nodes, tumour grade, serum BT, sPSA and patient age affected uPSA levels (Table 2). In fact, the only thing that seemed to affect uPSA levels was recurrent prostate

Table 2. Comparison of patients who had an elevated urinary prostate-specific antigen with those who did not

\begin{tabular}{|c|c|c|c|}
\hline \multirow[b]{2}{*}{ Variable } & \multicolumn{2}{|c|}{ No. (\%) of patients* } & \multirow[b]{2}{*}{$p$ value } \\
\hline & $\begin{array}{c}\text { Undetectable } \\
\text { uPSA, } \\
n=17\end{array}$ & $\begin{array}{c}\text { Detectable } \\
\text { uPSA, } \\
n=33\end{array}$ & \\
\hline $\begin{array}{l}\text { Age, median, } \\
\text { yr (IQR) }\end{array}$ & $64(62-66)$ & $68(60-72)$ & $0.11 \dagger$ \\
\hline \multicolumn{4}{|l|}{ TNM stage } \\
\hline $\mathrm{T} 1 \mathrm{~A} / \mathrm{B}$ & $1 \quad(6)$ & $2(6)$ & $0.79 \neq$ \\
\hline $\mathrm{T} 2$ & $10(59)$ & $15(45)$ & \\
\hline T3 & $6(35)$ & $16(48)$ & \\
\hline NO & $16(94)$ & $28(85)$ & $0.79 \ddagger$ \\
\hline $\mathrm{N} 1 / 2$ & 1 (6) & 5 (15) & \\
\hline \multicolumn{4}{|l|}{ Gleason score } \\
\hline 6 & $11(65)$ & 25 (76) & $0.54 \ddagger$ \\
\hline 7 & 3 (18) & 2 (6) & \\
\hline 8-10 & $3(18)$ & $6(18)$ & \\
\hline $\begin{array}{l}\text { Serum } \\
\text { testosterone, } \\
\text { nmol/L (IQR) }\end{array}$ & $1.0(0.5-1.6)$ & $1.0(0.8-1.6)$ & $0.39+$ \\
\hline \multicolumn{4}{|l|}{ Serum PSA } \\
\hline $\begin{array}{l}\text { Median, } \\
\text { mg/L (IQR) }\end{array}$ & $0(0-0)$ & $0(0-0)$ & $0.39+$ \\
\hline$>0$ & $2(12)$ & 6 (18) & \\
\hline PSA recurrence & $4(24)$ & $17(52)$ & $0.08 \ddagger$ \\
\hline \multicolumn{4}{|c|}{$\begin{array}{l}\text { IQR = interquartile range; PSA = prostatic-specific antigen; TNM = tumour, nodes, } \\
\text { metastases; uPSA = urinary prostatic-specific antigen. } \\
\text { *Unless otherwise indicated. } \\
\text { tWilcoxon rank-sum test. } \\
\text { fFisher exact test. }\end{array}$} \\
\hline
\end{tabular}


cancer. The 5- and 10-year sPSA recurrence-free survival rates of patients with detectable uPSA were $81 \%(95 \% \mathrm{Cl}$ $63-91)$ and $41 \%(95 \%$ Cl 22-60), respectively (Fig. 1). In contrast, the 5- and 10-year sPSA recurrence-free survival rates with undetectable uPSAs were both $77 \%(95 \% \mathrm{CI}$ 49-90), indicating a divergence in survival occurring at roughly 5 years postoperatively.

When we evaluated uPSA for its association with sPSA recurrence, we noted that the strength of association depended on sPSA level. This is demonstrated in Table 3, where it is shown that the odds of having an sPSA failure given a positive uPSA test was 4.5 if sPSA was undetectable, but was reduced to 2.6 if sPSA was detectable. The pooled

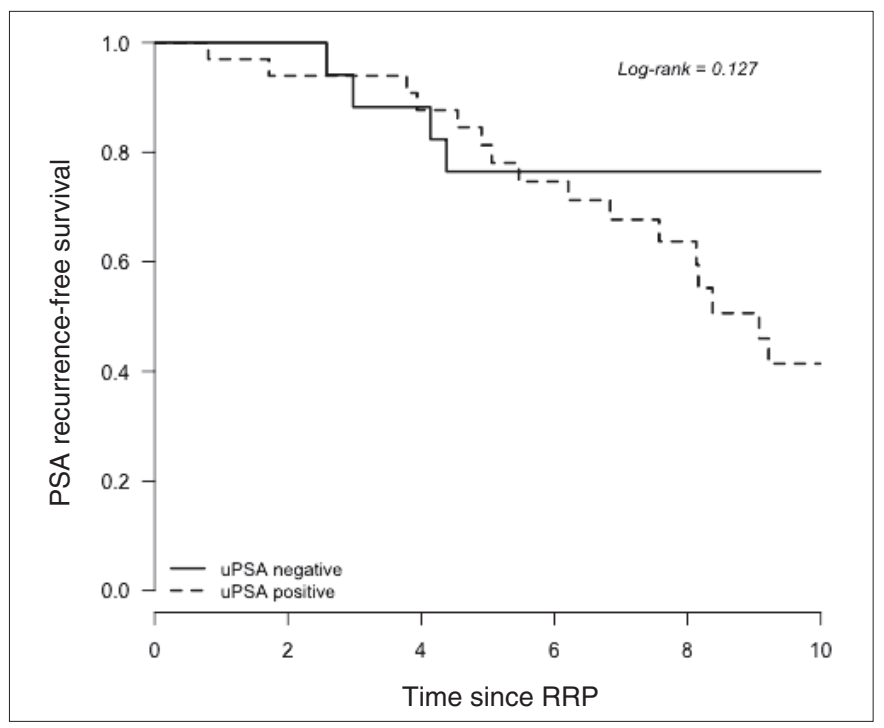

Fig. 1. Kaplan-Meier plot of serum prostate-specific antigen (PSA) recurrence-free survival given urinary PSA status. RRP = radical retropubic prostatectomy; uPSA = urinary prostate-specific antigen.

\begin{tabular}{|c|c|c|c|c|}
\hline \multirow[b]{2}{*}{ Recurrence } & \multicolumn{2}{|c|}{$\begin{array}{c}\text { Undetectable sPSA at } \\
\text { baseline }\end{array}$} & \multicolumn{2}{|c|}{$\begin{array}{c}\text { Detectable sPSA at } \\
\text { baseline }\end{array}$} \\
\hline & $\begin{array}{l}\text { Detectable } \\
\text { uPSA }\end{array}$ & $\begin{array}{c}\text { Undetectable } \\
\text { uPSA }\end{array}$ & $\begin{array}{l}\text { Detectable } \\
\text { uPSA }\end{array}$ & $\begin{array}{c}\text { Undetectable } \\
\text { uPSA }\end{array}$ \\
\hline Yes & 11 & 2 & 6 & 2 \\
\hline No & 16 & 13 & 0 & 0 \\
\hline Odds ratio & \multicolumn{2}{|r|}{4.5} & \multicolumn{2}{|c|}{$2.6+$} \\
\hline $\begin{array}{l}\text { Analysis of } \\
\text { uPSA* }\end{array}$ & \multicolumn{4}{|c|}{$4.21 \neq(95 \% \mathrm{Cl}, 0.89-19.77)$} \\
\hline \multicolumn{5}{|c|}{$\begin{array}{l}\mathrm{Cl}=\text { confidence interval; PSA = prostatic-specific antigen; } \text { sPSA = serum prostate- } \\
\text { specific antigen; uPSA = urinary prostate-specific antigen. } \\
\text { *Analysis confirming that having detectable uPSA quadrupled the risk of sPSA } \\
\text { recurrence, independent of whether sPSA was elevated or not. } \\
\text { tYates corrected odds ratio. } \\
\text { fMantel-Haenszel pooled odds ratio. }\end{array}$} \\
\hline
\end{tabular}

Mantel-Haenszel OR of 4.21 (95\% Cl 0.89-19.77, $p=0.12$ ) suggested that having a detectable uPSA quadrupled the risk of recurrence, independent of whether sPSA was elevated or not. Overall, the sensitivity of uPSA for detecting future sPSA recurrences was $81 \%$ and its specificity was $45 \%$. With the observed prevalence of sPSA recurrence of $42 \%$, the positive predictive value of the UPSA test was $52 \%$ and its negative predictive value was $76 \%$. The positive and negative diagnostic ORs were 1.47 and 0.42 , respectively.

\section{Discussion}

After the treatment of prostate cancer, a thorough and careful follow-up regimen must be ensured to detect cancer that has escaped initial treatment. At the present time, sPSA is the best tool we have to detect recurrent prostate cancer, regardless of whether the recurrence is confined to the prostatic fossa or metastatic in nature. In the present study, we assessed uPSA as a complementary method to detect recurrent prostate cancer after RRP. Based on previously published data, ${ }^{17}$ we hypothesized that uPSA might rise before sPSA and therefore provide an opportunity to initiate salvage treatment while the disease burden was still relatively low. Second, we felt that uPSA might be useful to distinguish cancer recurrences localized to the prostatic fossa from those that were metastatic. From previous work in our laboratory on the PSA physiology, we can add that the presence of PSA in the urine has to be by local secretion into the urethral lumen. In fact, PSA should not be found in the urine by glomerular filtration from a distant metastasis because the glomerulus does not normally allow the passage of proteins of the size and charge of PSA. Therefore, a local or regional recurrence not draining into the urethra (e.g., invasion of a regional lymph node) could lead to sPSA failure but not to uPSA detection. This distinction is clinically important because the treatment of a metastatic recurrence is very different from that of a local recurrence. Interestingly, among the patients with detectable UPSA and who presented sPSA failure, 6 of 7 responded to radiotherapy and also lowered their uPSA. All patients who received ADT saw their uPSA become undetectable. Undetectable uPSA associated with a significant decrease in BT was the main observation in a group of 38 patients receiving ADT (data not shown), confirming that production of uPSA by periurethral glands is under androgen control.' This observation could explain why 16 patients with detectable uPSA had sPSA that was still undetectable after 6 years; they had normal androgen levels with physiological periurethral gland PSA production but no evidence of disease. 
We observed that a positive uPSA test increased the risk of a subsequent biochemical failure by roughly 4 times. More generally, we noted that uPSA elevations detected in the year 2000 appeared to anticipate cancer recurrences that occurred from 2000-2006, a finding that was independent of the sPSA level in 2000. This suggests that UPSA may be a way of detecting recurrences in patients whose sPSA is low or normal, potentially years before prostate cancer recurrence would have otherwise been diagnosed. However, because of the small sample size in our study, the confidence limits for this OR span 1, indicating a result that is not statistically significant. Despite this, our data suggest that uPSA might be a promising new approach to screen for prostate cancer recurrences, a test that would cost very little to confirm in future studies given that it represents a minor adaptation of existing sPSA diagnostic technology. The next step would be a rigorous prospective trial involving more patients and comparing longitudinal (i.e., consecutive postop) sPSA measurements with longitudinal uPSA measurements.

Another important finding of our study was that uPSA levels were not dependent on the physiological levels of BT, patient age, or other factors that could have potentially altered test performance. This suggests that uPSA might be a diagnostic test that is relatively robust with respect to the variability commonly observed among and within patients, though more work is needed to prove this.

\section{Conclusion}

Urinary PSA could contribute to an early detection of local recurrences of prostate cancer after a radical prostatectomy. This clinically important distinction would allow the use of local therapy instead of systemic ADT. Larger prospective longitudinal studies are needed to determine whether uPSA testing is a clinically valid method of detecting local recurrences of prostate cancer.

From the *Division of Urology, Centre Hospitalier Universitaire de Québec (CHUQ), Université Laval, Québec, Que. and the †Division of Urology, Duke University Medical Center, Durham, NC

This article has been peer reviewed.
Competing interests: None declared.

\section{References:}

1. Graves HC, Sensabaugh GF, Blake ET. Postcoital detection of a male-specific semen protein. Application to the investigation of rape. N Engl J Med 1985;312:338-43.

2. Iwakiri J, Granbois K, Wehner N, et al. An analysis of urinary prostate specific antigen before and after radical prostatectomy: evidence for secretion of prostate specific antigen by the periurethral glands. J Urol 1993; 149:783-6.

3. Irani J. Urine PSA is not useful for detecting prostate cancer? Urology 1998;52:350-1.

4. Malavaud B, Salama $G$, Miedouge $M$, et al. Influence of digital rectal massage on urinary prostatespecific antigen: interest for the detection of local recurrence after radical prostatectomy. Prostate 1998; 34:23-8.

5. Bolduc S, Lacombe L, Naud A, et al. Urinary PSA: a potential useful marker when serum PSA is between $2.5 \mathrm{ng} / \mathrm{mL}$ and $10 \mathrm{ng} / \mathrm{mL}$. Can Urol Assoc J 2007; 1:377-81.

6. DeVere White RW, Meyers FJ, Soares SE, et al. Urinary prostate specific antigen levels: role in monitoring the response of prostate cancer to therapy. J Urol 1992; 147:947-51.

7. Breul J, Pickl U, Hartung R. Prostate-specific antigen in urine. Eur Urol 1994;26:18-21.

8. Pannek J, Rittenhouse HG, Evans $\mathrm{CL}$, et al. Molecular forms of prostate-specific antigen and human kallikrein 2 (hK2) in urine are not clinically useful for early detection and staging of prostate cancer. Urology 1997;50:715-21.

9. Tremblay J, Frenette G, Tremblay RR, et al. Excretion of three major prostatic secretory proteins in the urine of normal men and patients with benign prostatic hypertrophy or prostate cancer. Prostate 1987; 10:235-43.

10. Irani J, Millet C, Levillain $P$, et al. Serum-to-urinary prostate specific antigen ratio: its impact in distinguishing prostate cancer when serum prostate specific antigen level is 4 to $10 \mathrm{ng} . / \mathrm{ml}$. J Urol 1997; 157:185-8.

11. Pound CR, Partin AW, Epstein Jl, et al. Prostate-specific antigen after anatomic radical retropubic prostatectomy. Patterns of recurrence and cancer control. Urol Clin North Am 1997;24:395-406.

12. Partin AW, Pearson JD, Landis PK, et al. Evaluation of serum prostate-specific antigen velocity after radical prostatectomy to distinguish local recurrence from distant metastases. Urology 1994;43: 649-59.

13. Patel A, Dorey F, Franklin J, et al. Recurrence patterns after radical retropubic prostatectomy: clinical usefulness of prostate specific antigen doubling times and log slope prostate specific antigen. J Urol 1997; 158:1441-5.

14. Tremblay RR, Dubé JY. Plasma concentrations of free and non-TeBG bound testosterone in women on oral contraceptives. Contraception 1974;10:599-605.

15. R Development Core Team. The R project for statistical computing. R Foundation for Statistical Computing; 2008. Available at: www.R-project.org (accessed 2009 Feb 18).

16. Mantel N, Haenszel W. Statistical aspects of the analysis of data from retrospective studies of disease. J Natl Cancer Inst 1959:22:719-48.

17. Takayama TK, Krieger JN, True LD, et al. Recurrent prostate cancer despite undetectable prostate specific antigen. J Urol 1992;148:1541-2.

Correspondence: Dr. Stéphane Bolduc, Division of Urology, Centre Hospitalier Universitaire de Québec (CHUQ, CHUL), 2705 Boul. Laurier, R-1742, Québec, QC GIV 4G2; fax 418 654-2137; sbolduc_2002@yahoo.ca 